\title{
Association of the 1q25 Diabetes-Specific Coronary Heart Disease Locus With Alterations of the $\gamma$-Glutamyl Cycle and Increased Methylglyoxal Levels in Endothelial Cells
}

\author{
Caterina Pipino,1,2,3 Hetal Shah,1,2 Sabrina Prudente, ${ }^{4}$ Natalia Di Pietro, ${ }^{3}$ Lixia Zeng, 5 Kyoungmin Park,1,2 \\ Vincenzo Trischitta, ${ }^{6,7}$ Subramanian Pennathur, ${ }^{5}$ Assunta Pandolfi, ${ }^{3}$ and Alessandro Doria ${ }^{1,2}$
}

Diabetes 2020;69:2206-2216 | https://doi.org/10.2337/db20-0475

\begin{abstract}
A chromosome 1q25 variant (rs10911021) has been associated with coronary heart disease (CHD) in type 2 diabetes. In human umbilical vein endothelial cells (HUVECs), the risk allele " $C$ " is associated with lower expression of the adjacent gene GLUL encoding glutamine synthase, converting glutamic acid to glutamine. To further investigate the mechanisms through which this locus affects CHD risk, we measured 35 intracellular metabolites involved in glutamic acid metabolism and the $\gamma$-glutamyl cycle in 62 HUVEC strains carrying different rs10911021 genotypes. Eight metabolites were positively associated with the risk allele (17-58\% increase/allele copy, $P=0.046-0.002$ ), including five $\gamma$-glutamyl amino acids, $\beta$-citryl-glutamate, $N$-acetylaspartyl-glutamate, and ophthalmate-a marker of $\gamma$-glutamyl cycle malfunction. Consistent with these findings, the risk allele was also associated with decreased glutathione-to-glutamate ratio $(-9 \%, P=0.012)$, decreased $S$-lactoylglutathione $(-41 \%, P=0.019)$, and reduced detoxification of the atherogenic compound methylglyoxal $(+54 \%, P=0.008)$. GLUL downregulation by shRNA caused a $40 \%$ increase in the methylglyoxal level, which was completely prevented by glutamine supplementation. In summary, we have identified intracellular metabolic traits associated with the 1q25 risk allele in HUVECs, including impairments of the $\gamma$-glutamyl cycle and methylglyoxal detoxification. Glutamine supplementation abolishes the latter abnormality,
\end{abstract}

${ }^{1}$ Research Division, Joslin Diabetes Center, Boston, MA

2Department of Medicine, Harvard Medical School, Boston, MA

${ }^{3}$ Department of Medical, Oral and Biotechnological Sciences, Center for Advanced Studies and Technology - CAST (ex CeSI-MeT), University G. d'Annunzio of ChietiPescara, Chieti, Italy

${ }^{4}$ Research Unit of Metabolic and Cardiovascular Diseases, Fondazione IRCCS Casa Sollievo della Sofferenza, San Giovanni Rotondo, Italy

${ }^{5}$ Division of Nephrology, Department of Medicine, University of Michigan, Ann Arbor, Ml ${ }^{6}$ Research Unit of Diabetes and Endocrine Diseases, Fondazione IRCCS Casa Sollievo della Sofferenza, San Giovanni Rotondo, Italy

${ }^{7}$ Department of Experimental Medicine, Sapienza University, Rome, Italy suggesting that such treatment may prevent CHD in 1q25 risk allele carriers.

Despite improvements in glycemic control and other preventive therapies, individuals with type 2 diabetes continue to experience an increased burden of cardiovascular complications such as coronary heart disease (CHD) (1). In order to change the status quo, new interventions are needed that specifically target the mechanisms linking the diabetic milieu to vascular damage. Our strategy to gain insights into these mechanisms and identify novel targets for preventive therapies has been to study the genetic factors that modulate cardiovascular risk among people with type 2 diabetes (2).

Following this approach, we have discovered a locus on chromosome 1q25 that is associated with CHD, with genome-wide significance, in multiple sets of patients with type 2 diabetes (3). Subsequent studies have confirmed this finding in other populations with type $2 \mathrm{di}$ abetes $(4,5)$. This CHD locus is placed in the region of the GLUL gene, which codes for glutamate-ammonia ligase (also known as glutamine synthase) catalyzing the conversion of glutamic acid to glutamine (6). In the original report, the risk allele of the lead variant (rs10911021) was associated with decreased GLUL expression in endothelial cells and with a lower pyroglutamic-to-glutamic acid ratio in plasma, suggesting an impairment of glutamic
Corresponding author: Alessandro Doria, alessandro.doria@joslin.harvard.edu Received 5 May 2020 and accepted 3 July 2020

This article contains supplementary material online at https://doi.org/10.2337/ figshare.12616442.

(C) 2020 by the American Diabetes Association. Readers may use this article as long as the work is properly cited, the use is educational and not for profit, and the work is not altered. More information is available at https://www.diabetesjournals .org/content/license. 
acid metabolism and the $\gamma$-glutamyl cycle, of which pyroglutamic acid is an intermediate, as a possible mechanism underlying the association with CHD (3). The $\gamma$-glutamyl cycle is responsible for the generation of glutathione-a natural antioxidant playing a critical role in the protection from free radicals and other reactive compounds (7) —-whose deficit may predispose to increased oxidative stress and accelerated atherosclerosis $(7,8)$.

The goal of the current study was to seek further support for this hypothesis by analyzing the association at the cellular level between the 1q25 lead variant (rs10911021) and metabolites involved in glutamic acid metabolism and the $\gamma$-glutamyl cycle in a unique collection of human umbilical vein endothelial cells (HUVECs) isolated from a large group of newborns and therefore allowing the study of the impact of natural genetic variation on cellular functions. Our results support the hypothesis of an association between the 1q25 locus and alternations in the $\gamma$-glutamyl cycle and point to a reduced glutathione-mediated detoxification of methylglyoxal-a precursor of advanced glycation end products (AGEs) - as a possible mechanism linking these metabolic changes to the increased CHD risk experienced by $1 \mathrm{q} 25$ risk allele carriers with type 2 diabetes.

\section{RESEARCH DESIGN AND METHODS}

\section{HUVEC Preparation and Culture}

The study was performed on HUVECs isolated from the umbilical cords of 62 newborns delivered by randomly selected, healthy Caucasian mothers between the 36th and the 40th gestational week at the Hospital of Chieti and Pescara (Italy). HUVECs were isolated from the umbilical cords by extraction with $1 \mathrm{mg} / \mathrm{mL}$ collagenase $1 \mathrm{~A}$ at $37^{\circ} \mathrm{C}$, as previously described (9). All procedures were in agreement with the ethical standards of the local Institutional Committee on Human Experimentation (reference number: 1879/09COET) (University G. d'Annunzio of Chieti-Pescara, Chieti, Italy) and with the Declaration of Helsinki ethical principles. After approval of the protocol by the Institutional Review Board, signed informed consent was obtained from each participating subject.

For this study, HUVECs were grown on $1.5 \%$ gelatincoated tissue culture plates (Sigma-Aldrich) in complete low-glucose ( $1 \mathrm{~g} / \mathrm{L})$ culture medium composed of DMEM (provided by Joslin Media Core) supplemented with $20 \%$ FBS (Gibco by Life Technologies), 200 units/mL penicillin, $200 \mu \mathrm{g} / \mathrm{mL}$ streptomycin (Sigma-Aldrich), $10 \mu \mathrm{g} / \mathrm{mL}$ heparin (Stem Cell Technologies), and $50 \mu \mathrm{g} / \mathrm{mL}$ endothelial cells growth factor (Alfa Aeser). HUVECs were maintained in incubator at $37^{\circ} \mathrm{C}$ and in a humidified atmosphere of $5 \%$ $\mathrm{CO}_{2} / 95 \%$ air and subcultured using $0.05 \%$ trypsin $/ 0.02 \%$ EDTA. Cells between the third and seventh passages were used in all experiments.

\section{Genotyping}

DNA was extracted from HUVECs by standard methods. Genotyping of the rs10911021 variant was performed by means of a custom TaqMan assay (Life Technologies, Foster City, CA) on a 7900HT platform (Applied Biosystems, Foster City, CA). Genotyping quality was tested by including six blinded duplicate samples in each 96-well assay. The average agreement rate of the duplicate samples was $>99 \%$.

\section{Metabolomic Profiling \\ Cell Culture}

HUVEC s at $60-70 \%$ confluence were incubated with media containing 10\% FBS and $5.5 \mathrm{mmol} / \mathrm{L}$ D-glucose (basal condition, low glucose) or $25 \mathrm{mmol} / \mathrm{L}$ D-glucose (high glucose). After $48 \mathrm{~h}$ of treatment, cells were trypsinized, transferred to a $50-\mathrm{mL}$ polypropylene Falcon tube, and centrifuged at 1,200 rpm for $10 \mathrm{~min}$. Supernatants were discarded and cell pellets gently resuspended in PBS and transferred to prelabeled 2.0-mL polypropylene tubes. Cell suspensions were centrifuged (1,200 rpm for $10 \mathrm{~min})$ and all supernatants carefully removed. Cell pellets were immediately stored at $-80^{\circ} \mathrm{C}$ until all biological replicates were collected and shipped to Metabolon (Durham, NC) for metabolomic profiling.

\section{Metabolite Measurements}

The current study considered 35 metabolites involved in glutamic acid metabolism, the $\gamma$-glutamyl cycle, and glutathione metabolism that were extracted from a larger Metabolon panel of 597 metabolites according to the prespecified hypothesis of an impact of the 1q25 locus on these metabolic pathways. These 35 metabolites included all those whose "Sub-pathway" field in the Metabolon report was labeled as "glutathione metabolism" $(n=9)$, "glutamate metabolism" $(n=13)$, or " $\gamma$-glutamyl amino acid" ( $n=10)$, plus cysteine and glycine (the two amino acids that are used together with glutamic acid to synthesize glutathione) and $\alpha$-ketoglutarate, a Krebs cycle intermediate that is in direct equilibrium with glutamic acid through amination/deamination by glutamate dehydrogenase. Samples were prepared by Metabolon using the automated MicroLab STAR system from Hamilton Company. Several recovery standards were added before the first step in the extraction process for quality control purposes.

To remove proteins, dissociate small molecules bound to proteins or trapped in the precipitated protein matrix, and to recover chemically diverse metabolites, proteins were precipitated with methanol under vigorous shaking for 2 min (GenoGrinder 2000; Glen Mills), followed by centrifugation. The resulting extracts were divided into five fractions: two for analysis by two separate reversephase (RP)/ultra high-performance liquid chromatography (UPLC)-tandem mass spectrometry (MS/MS) methods with positive ion mode electrospray ionization (ESI), one for analysis by RP/UPLC-MS/MS with negative ion mode 
ESI, one for analysis by hydrophilic interaction chromatography/UPLC-MS/MS with negative ion mode ESI, and one reserved for backup. Samples were placed briefly on a TurboVap (Zymark) to remove the organic solvent. Extracts were stored overnight under nitrogen before preparation for analysis.

\section{Quality Assurance/Quality Control}

Several types of controls were analyzed along with the experimental samples: a pooled matrix sample generated by taking a small volume of each experimental sample served as a technical replicate throughout the analyses, extracted water samples served as process blanks, and a cocktail of quality control standards spiked into every analyzed sample allowed for instrument performance monitoring and aided chromatographic alignment. Instrument variability was determined by calculating the median relative SD (RSD) for the standards that were added to each sample before injection into the mass spectrometers. Overall process variability was determined by calculating the median RSD for all endogenous metabolites (i.e., noninstrument standards) present in $100 \%$ of the pooled matrix samples. Experimental samples were randomized across the platform run, with quality control samples spaced evenly among the injections.

\section{Western Blotting}

When $70 \%$ confluence was reached, HUVECs were cultured in basal conditions ( $5.5 \mathrm{mmol} / \mathrm{L}$ D-glucose) or treated with high glucose ( $25 \mathrm{mmol} / \mathrm{L}$ D-glucose) for $48 \mathrm{~h}$. Briefly, cells were washed with cold PBS and lysed in RIPA buffer (Sigma-Aldrich), with the addition of protease and phosphatase inhibitors (cat. P8340 and cat. P5726; SigmaAldrich). The protein concentration of the lysates was determined using the Pierce BCA Protein Assay Kit (Thermo Fisher Scientific), and $30 \mu \mathrm{g}$ of each sample was separated by SDS-PAGE and transferred to polyvinylidene fluoride membrane (Bio-Rad, Hercules, CA). Membranes were blocked with $5 \%$ nonfat milk, followed by immunoblotting with the primary antibody against rabbit antiGLUL polyclonal antibody (1:2,000) (Bethyl) overnight at $4^{\circ} \mathrm{C}$, followed by rabbit $(1: 2,000)$ (Cell Signaling Technology) horseradish peroxidase-conjugated secondary antibody $(1: 2,000)$ (Santa Cruz Biotechnology). Immune complexes were visualized by means of the ECL Plus detection reagent (Thermo Scientific), and data were processed and quantified by ImageJ software. Protein densities were divided by $\beta$-actin densities (mouse monoclonal anti- $\beta$-actin, 1:4,000), and the resulting ratio was considered as an index of GLUL expression in arbitrary units.

\section{Transduction of HUVECs With shRNA Lentiviral Particles}

shRNA lentiviral particles, including a nontarget control (CSHCTR001-1-LVRH1GP) and several shRNA targeting GLUL (CS-HSH065270-31-LVRH1GP-01, CS-HSH06527032-LVRH1GP-01, CS-HSH065270-33-LVRH1GP-01, and
CS-HSH065270-34-LVRH1GP-01) were purchased from GeneCopoeia. The nontarget control contained a scrambled sequence (5'-GCTTCGCGCCGTAGTCTTA-3') not targeting any known human gene, whereas lentiviruses targeting the GLUL gene contain the following sequences: 5'-GCAAGTTCCCACTTAAATAAA-3', 5' -GGCTCTAGTACTTTA CAGTCT-3', 5'-CCTGTAAACGGATAATGGACA-3', and 5'-GCACGTGTCTTCTCAATGAAA-3' .

Transductions were performed according to the manufacturer's protocol. Briefly, $0.3 \times 10^{6}$ HUVECs were seeded into a six-well plate and incubated for 2 days at $37^{\circ} \mathrm{C}$ in a $5 \% \mathrm{CO}_{2}$ incubator. Cells were infected overnight with control (scramble) and GLUL lentiviral particles at multiplicities of infection of $1,3,5$, and 10 in the presence of $8 \mu \mathrm{g} / \mathrm{mL}$ Polybrene Infection/Transfection reagent (Millipore). Negative control wells included cells not infected with virus and with the addition of Polybrene. All cells were incubated in fresh complete medium for 2 more days for a total of $72 \mathrm{~h}$ since the beginning of transduction. Reduction of GLUL protein expression was confirmed by Western blotting (see above).

For some experiments, after transduction with GLUL shRNA, cells were supplemented with glutamine (SigmaAldrich) at a final concentration of $10 \mathrm{mmol} / \mathrm{L}$ when the medium was changed.

\section{Evaluation of Oxidized Tyrosines}

Cells were plated in 100-mm Petri dishes at a concentration of $1.5 \times 10^{6}$. When $70 \%$ confluence was reached, cells were cultured in basal conditions ( $5.5 \mathrm{mmol} / \mathrm{L}$ D-glucose) or treated with high glucose ( $25 \mathrm{mmol} / \mathrm{L}$ D-glucose) for $48 \mathrm{~h}$. Cells were washed twice with PBS, and then $1 \mathrm{~mL}$ of PBS supplemented with $0.005 \%$ butylated hydroxytoluene (BHT) was added to each plate. Cells were scraped, collected in cryotubes, and immediately stored at $-80^{\circ} \mathrm{C}$. As markers of oxidative stress, protein-bound oxidized tyrosine moieties, 3-nitrotyrosine, 3-chlorotyrosine, and $\mathrm{o}, \mathrm{o}^{\prime}$-dityrosine were quantified in cell extracts using isotopedilution high-performance liquid chromatography-ESIMS/MS, as described previously (10). Briefly, the cell lysates were subjected to protein precipitation with icecold trichloroacetic acid $(10 \% \mathrm{vol} / \mathrm{vol})$, delipidated with water/methanol/water-washed diethyl ether (1:3:7 vol/ $\mathrm{vol} / \mathrm{vol})$, and known amounts of isotopically labeled internal standards ${ }^{13} \mathrm{C}_{6}$-tyrosine, and ${ }^{13} \mathrm{C}_{6}$-3-nitrotyrosine, ${ }^{13} \mathrm{C}_{6}$-chlorotyrosine, and ${ }^{13} \mathrm{C}_{12}$-o,o'-dityrosine were added. These preparations were further hydrolyzed at $110^{\circ} \mathrm{C}$ for $24 \mathrm{~h}$ in $4 \mathrm{~mol} / \mathrm{L}$ methanesulfonic acid solution saturated with $1 \%$ benzoic acid and subject to solid-phase extraction. The oxidized amino acids were quantified by high-performance liquid chromatography-ESI-MS/MS with multiple reactions monitoring by integrating peak areas of the labeled standards and the analytes. The levels of the oxidized amino acids were then normalized to the precursor amino acid tyrosine content. The levels of 
oxidized tyrosine products are expressed as the ratio of the oxidized product over the total tyrosine.

\section{Measurement of Methylglyoxal Levels}

Methylglyoxal protein adducts in 62 HUVEC protein lysates and in the cultures where GLUL was downregulated were quantified by the OxiSelect Methylglyoxal enzyme immunoassay (cat. no. STA-811; Cell Biolabs, San Diego, CA) according to the manufacturer's protocol. Briefly, after protein concentrations were determined with the Pierce BCA Protein Assay Kit (Thermo Fisher Scientific), samples were diluted with $1 \times \mathrm{PBS}$ to a volume sufficient to load $50 \mu \mathrm{g}$ of total protein per well. Methylglyoxal-BSA standards or protein samples were absorbed onto a 96-well plate overnight at $4^{\circ} \mathrm{C}$ and then exposed to an antimethylglyoxal-specific monoclonal antibody for $1 \mathrm{~h}$ at room temperature, followed by exposure to a horseradish peroxidase-conjugated secondary antibody. The methylglyoxal protein adduct content in experimental samples was determined by comparing its absorbance with that of a methylglyoxal-BSA standard curve.

\section{Statistical Analysis}

The measured values of metabolites obtained from Metabolon were normalized in terms of raw area counts, and these values were then rescaled to set the median equal to 1 . Metabolites below the detection limit (Supplementary Table 1) were imputed with the minimum value. Values for each metabolite were then normalized by the median values from each HUVEC sample. Each median-scaled metabolite was then $\log _{2}$ transformed to approximate the normal distribution. In the present analysis, 35 metabolites involved in glutamic acid metabolism, the $\gamma$-glutamyl cycle, and glutathione metabolism were extracted from a larger Metabolon panel of 597 metabolites, as described above under METABOLITE MEASUREMENTS. The association between each metabolite (dependent variable) and rs10911021 (independent variable) was analyzed separately in the low- and high-glucose experiments by linear mixed-effects models, with the single nucleotide polymorphism (SNP) considered as a fixed effect according to an additive model (i.e., as number of copies of the risk allele $C$ ) and the Metabolon analytical block as random effects. Linear mixed-effects models were similarly used for the combined analysis of low- and high-glucose experiments, including glucose and rs10911021 as fixed effects and HUVEC ID and Metabolon analytical block as random effects. The modifying effects of glucose on the SNP's association with the metabolites were assessed by incorporating SNP $\times$ glucose interaction terms in the main effects mixed models. A similar approach (linear regression or mixed linear models, as appropriate) was applied to the analysis of other variables measured outside of the Metabolon platform, such as GLUL protein levels, and methylglyoxal and oxidized tyrosine cell contents. Analyses were normalized by "assay batch" if data were generated in different experiments. All analyses were conducted in SAS 9.4 software (SAS Institute, Cary, NC). Volcano plots were generated in R software (R Core Team, Vienna, Austria). Since the study examined a prespecified hypothesis and most of the metabolites were correlated with each other, an unadjusted $P$ value of $<0.05$ was considered as significant.

\section{Data Resource and Availability}

The data sets generated during and/or analyzed during the current study are available from the corresponding author upon request.

\section{RESULTS}

\section{HUVEC Strain Characteristics}

The association between rs10911021 genotype and intracellular levels of metabolites involved in glutamic acid metabolism and the $\gamma$-glutamyl cycle was evaluated in primary HUVEC strains isolated from 62 newborns. We found 30 of these HUVECs were homozygous for the rs10911021 $C$ allele $(C / C), 21$ were heterozygous $(C / T)$, and 11 were homozygous for the $\mathrm{T}$ allele $(\mathrm{T} / \mathrm{T})$. Cells were
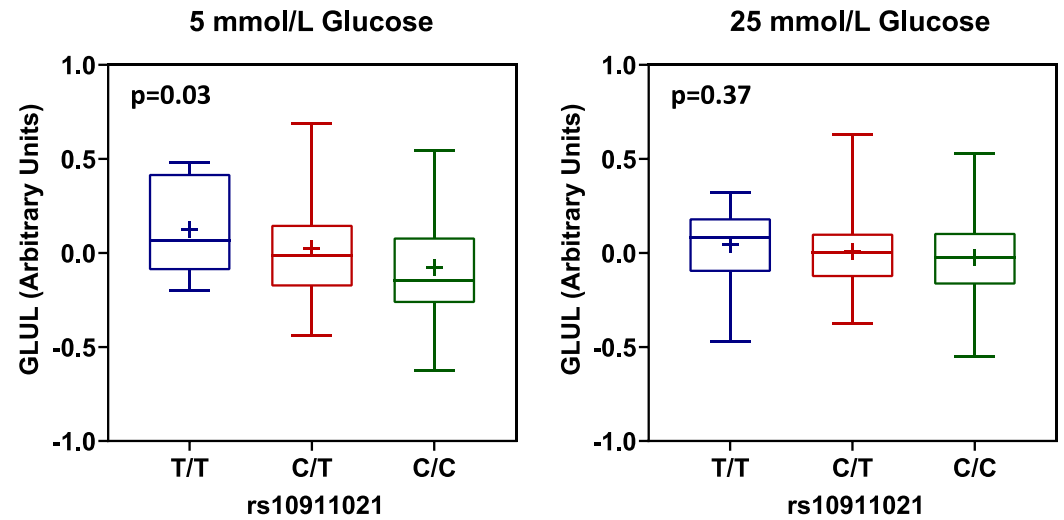

Figure 1-GLUL protein levels in HUVECs carrying different rs10911021 genotypes. Distributions of GLUL levels relative to $\beta$-actin, expressed as arbitrary units and normalized by assay batch, are shown for $62 \mathrm{HUVECs} \mathrm{exposed} \mathrm{to} 5 \mathrm{mmol} / \mathrm{L}$ or $25 \mathrm{mmol} / \mathrm{L}$ glucose. Boxes correspond to the interquartile range, the line within the box to the median, and the cross to the mean. Whiskers correspond to the maximum and minimum values. 
grown in low $(5.5 \mathrm{mmol} / \mathrm{L})$ and high $(25 \mathrm{mmol} / \mathrm{L})$ glucose. Consistent with the previously reported association between rs10911021 and GLUL expression levels in HUVECs, cells carrying the $C / C$ genotype had $36 \%$ lower GLUL protein levels than those with the $\mathrm{T} / \mathrm{T}$ genotype when grown in low glucose, with heterozygous cells having intermediate levels $(P=0.03)$ (Fig. 1). A similar trend was observed in cells grown in high glucose, although the difference among genotypes was smaller and did not reach statistical significance (Fig. 1).

\section{Effect of rs 10911021 Genotype on Metabolites Related to Glutamic Acid Metabolism and the $\gamma$-Glutamyl Cycle}

A total of 35 metabolites were included in this targeted metabolomic analysis, including 14 related to glutamic acid metabolism and 21 related to the $\gamma$-glutamyl cycle and glutathione metabolism (Supplementary Tables 1 and 2). As shown by the volcano plots in Fig. 2, five metabolites ( $\gamma$-glutamyl-threonine, $\gamma$-glutamyl-leucine, $\gamma$-glutamylisoleucine, $\gamma$-glutamyl-valine, and $\beta$-citryl-glutamate) showed a significant association $(P<0.05)$ with the genotype in both low and high glucose, two (ophthalmate and $N$-acetyl-aspartyl-glutamate) only in low glucose, and another two ( $S$-lactoyl-glutathione and $\gamma$-glutamyl-cysteine) only in high glucose (Fig. 2 and Supplementary Table 2). In a joint analysis of low- and high-glucose experiments, all the above metabolites were significantly associated with the rs10911021 genotype, with no evidence of a genotype $\times$ glucose interaction (Fig. 2 and Supplementary Table 2). All nine metabolites showed a significant correlation with one or more of the other metabolites in the group (Supplementary Table 3). Eight of the metabolites (the five $\gamma$-glutamyl amino acids, $\beta$-citryl-glutamate, ophthalmate, and $\mathrm{N}$-acetyl-aspartyl-glutamate) were positively associated with the $C$ allele (i.e., their levels rose with the increasing dosage of this allele) (Fig. 3). The strongest effect was observed for $\gamma$-glutamyl-threonine ( $+58 \%$ per copy of the $C$ allele), followed by ophthalmate $(+36 \%)$, and $\gamma$-glutamyl-valine $(+35 \%)$ (Fig. 3 and Supplementary Table 2). One of the nine metabolites $(S$ lactoyl-glutathione) showed instead a negative association with rs10911021. This was especially evident in high glucose conditions, in which cells carrying the $C$ allele had $80 \%$ lower levels of S-lactoyl-glutathione than T/T cells (Fig. 3 and Supplementary Table 2). These associations were not affected by adjustment for GLUL protein levels.

No association was detected between rs10911021 and intracellular levels of glutamate or glutamine (the substrate and the product, respectively, of the enzymatic reaction catalyzed by GLUL) or with glutathione (GSH, the product of the $\gamma$-glutamyl cycle) in either low or high glucose or in a joint analysis of the two conditions (Supplementary Table 2 and Supplementary Fig. 1). However, in both low- and high-glucose conditions, the ratio between GSH and glutamate levels was inversely correlated with the dosage of the $C$ allele (Fig. $4 A$ ), with an average $9 \%$ decrease per allele copy. Also, in both conditions, the GSH-
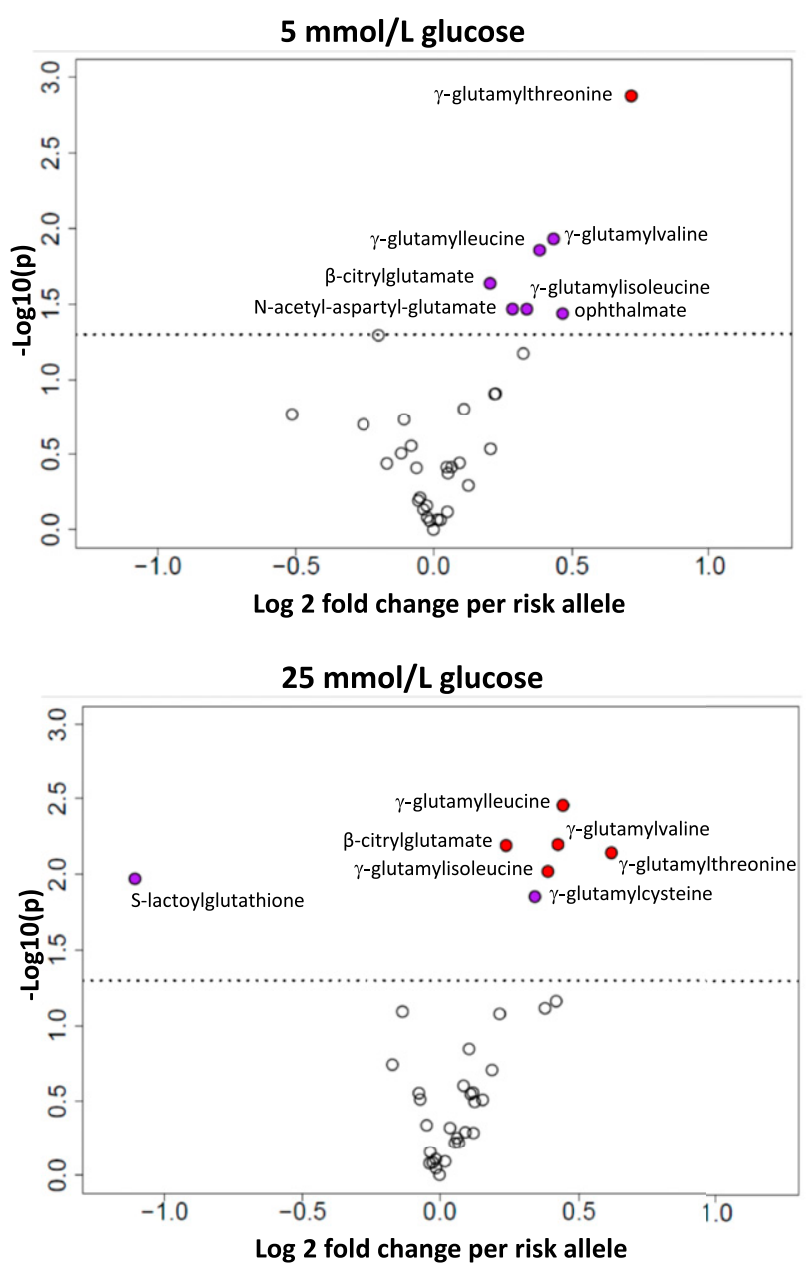

$5 \mathrm{mmol} / \mathrm{L}$ and $25 \mathrm{mmol} / \mathrm{L}$ glucose combined

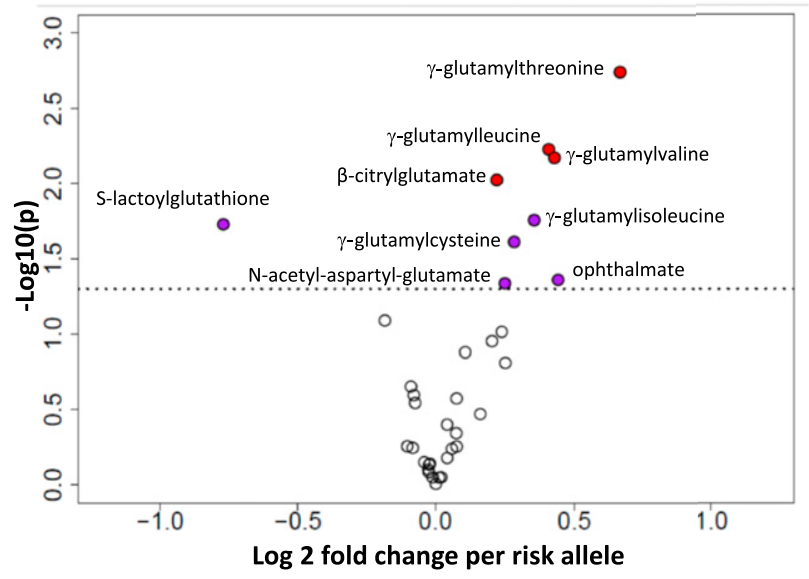

Figure 2-Association between the 1q25 (rs10911021) genotype and 35 metabolites related to glutamate metabolism and the $\gamma$-glutamyl cycle in HUVECs. Association results are shown for 62 HUVECs exposed to $5 \mathrm{mmol} / \mathrm{L}$ or $25 \mathrm{mmol} / \mathrm{L}$ glucose and in a joint analysis of the two glucose conditions. The association with the rs10911021 genotype is expressed as $\log _{2}$ fold change per allele copy ( $x$-axis), and significance is presented as $-\log _{10} P$ value ( $y$-axis). The dotted line indicates the $P=0.05$ threshold. Significant metabolites of interest are indicated by red $(P<0.01)$ and purple $(P<0.05)$ circles. 
$\gamma$-glutamyl-threonine

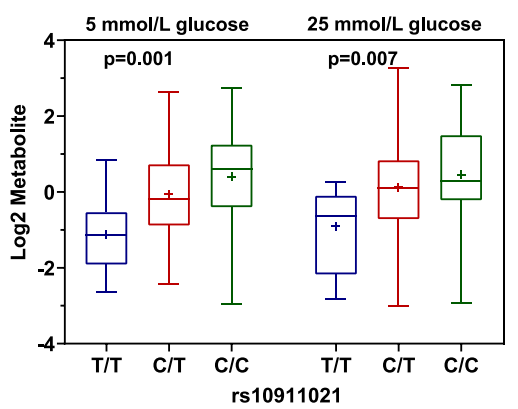

$\beta$-citryl-glutamate

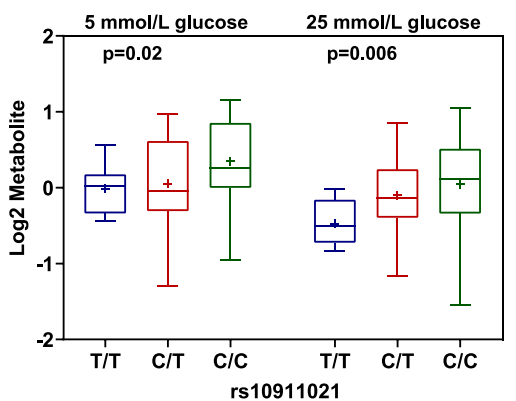

$\gamma$-glutamyl-cysteine

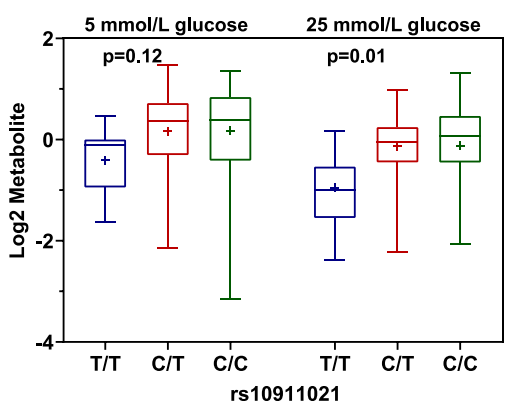

$\gamma$-glutamyl-leucine

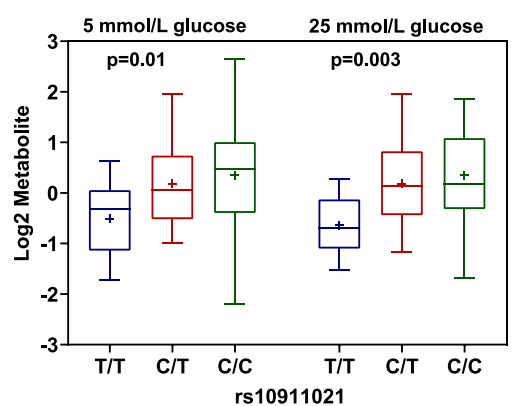

$\gamma$-glutamyl-isoleucine

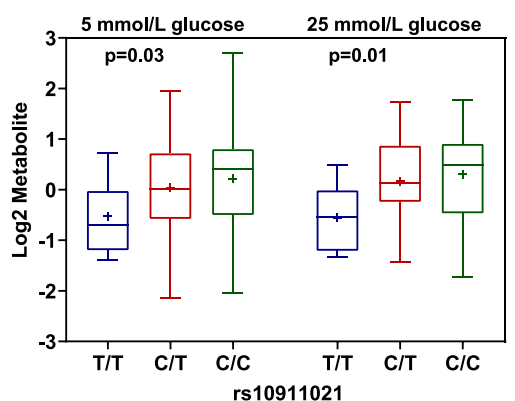

Ophthalmate

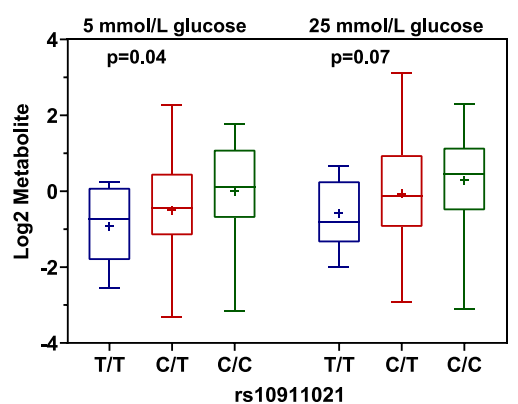

$\gamma$-glutamyl-valine

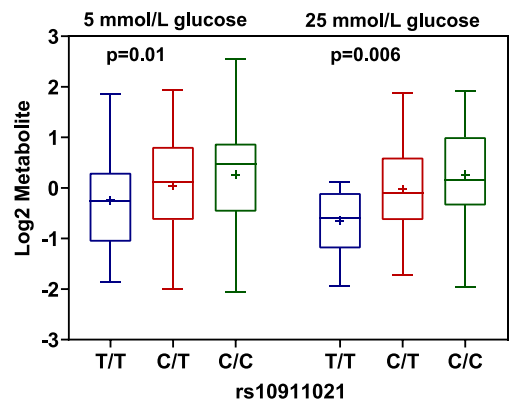

S-lactoyl-glutathione

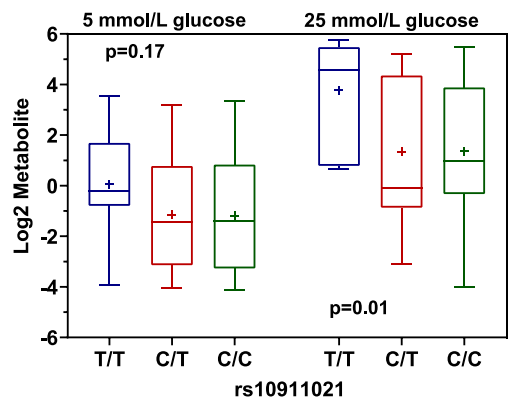

$\mathrm{N}$-acetyl-aspartyl-glutamate (NAAG)

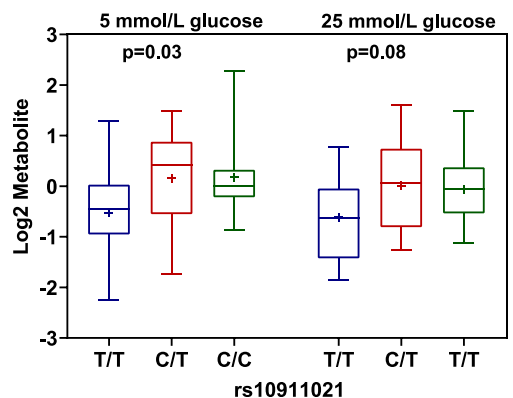

Figure 3-Association between 1q25 genotype and metabolite levels in HUVECs. Distributions according to rs10911021 genotype in $62 \mathrm{HUVECs} \mathrm{exposed} \mathrm{to} 5 \mathrm{mmol} / \mathrm{L}$ or $25 \mathrm{mmol} / \mathrm{L}$ glucose are shown for $\gamma$-glutamyl-threonine, $\gamma$-glutamyl-leucine, $\gamma$-glutamyl-valine, $\beta$-citrylglutamate, $\gamma$-glutamyl-isoleucine, $S$-lactoyl-glutathione, $\gamma$-glutamyl-cysteine, ophthalmate, and $N$-acetyl-aspartyl-glutamate (NAAG). Boxes correspond to the interquartile range, the line within the box to the median, and the cross to the mean. Whiskers correspond to the maximum and minimum values.

to-glutamate ratio showed a highly significant inverse correlation with $\gamma$-glutamyl-isoleucine, $\gamma$-glutamyl-leucine, $\gamma$-glutamyl-valine, $\gamma$-glutamyl-threonine, and ophthalmate (Fig. $4 B$ and Supplementary Table 4).

\section{Effect of rs10911021 Genotype on Methylglyoxal Levels}

$S$-lactoyl-glutathione-the only metabolite showing a negative association with rs10911021 allele C-originates in part from the glutathione-mediated detoxification of the reactive compound methylglyoxal, a glycolysis side product. Methylglyoxal is a precursor of AGEs, which have been implicated in the etiology of the vascular complications of diabetes (11). Since $S$-lactoyl-glutathione levels were lower in cells carrying the $C$ allele, (Fig. 3), we hypothesized that the $C$ allele was associated with defective methylglyoxal detoxification. Indeed, each additional copy of the $C$ allele was associated with a $69 \%(P=0.003)$ and $45 \%(P=0.03)$ increase in methylglyoxal levels in cultures exposed to low and high glucose, respectively (Fig. 5A). Compared with HUVECs with the T/T genotype, cells homozygous for the $C$ allele had 2.8-fold higher methylglyoxal levels in low glucose and 2.1-fold higher levels in high glucose, with heterozygous cells having intermediate levels in both conditions (Fig. 5A). The rs10911021 genotype had little or no effect on other intracellular markers of oxidative stress such as oxidized tyrosines. The only significant association was with 3-chloro-tyrosine levels in $25 \mathrm{mmol} / \mathrm{L}$ glucose, but the effect of the genotypes with this metabolite was much smaller than that on methylglyoxal levels (Supplementary Fig. 2).

To assess whether the lower GLUL levels associated with the allele $C$ were responsible for the higher methylglyoxal levels observed in cells carrying this allele, GLUL protein levels were downregulated by means of shRNA 
A

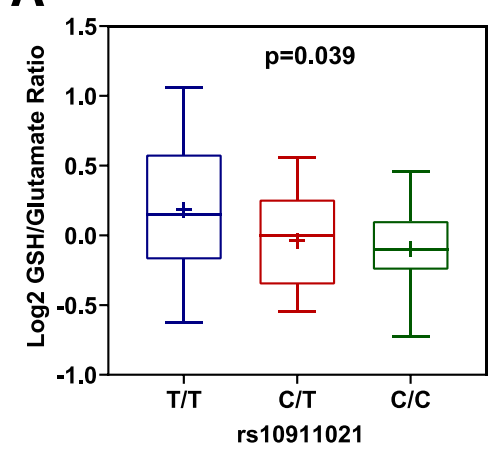

B $\quad 5 \mathrm{mmol} / \mathrm{L}$ glucose

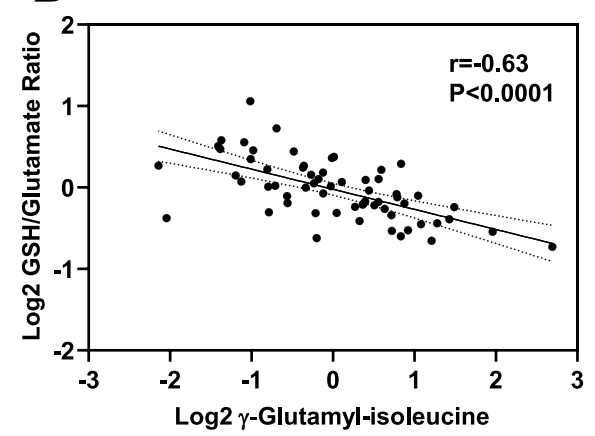

$5 \mathrm{mmol} / \mathrm{L}$ glucose

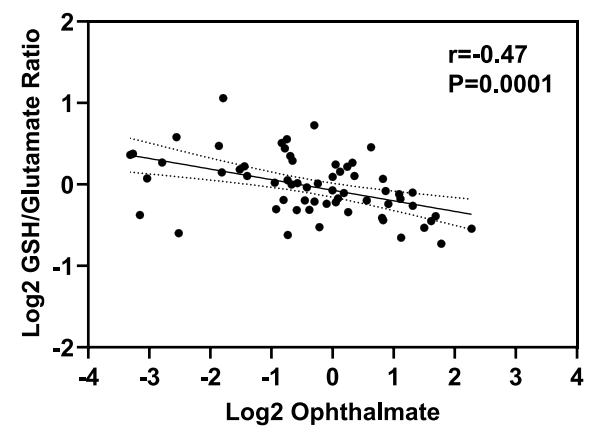

$25 \mathrm{mmol} / \mathrm{L}$ glucose

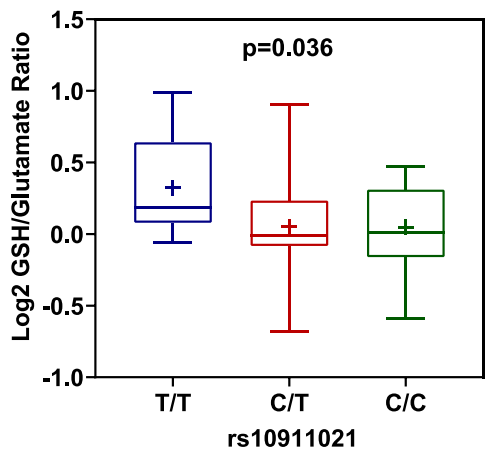

$25 \mathrm{mmol} / \mathrm{L}$ glucose

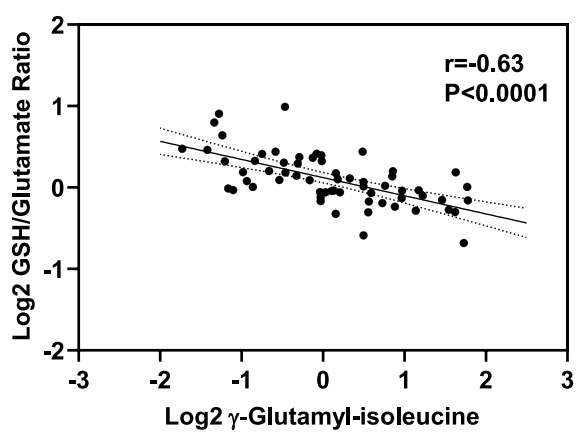

$25 \mathrm{mmol} / \mathrm{L}$ glucose

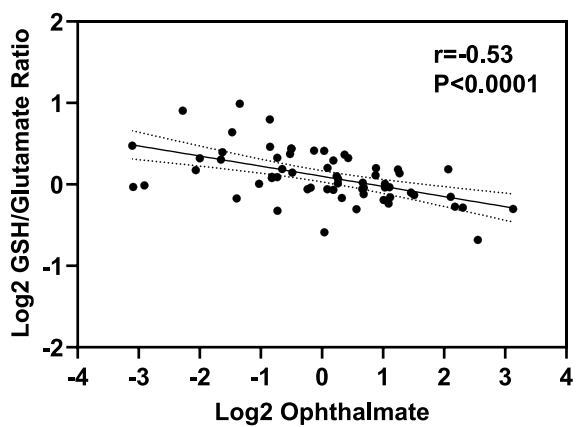

Figure 4-GSH-to-glutamate ratio in HUVECs. A: Distributions of $\log _{2}$ GSH-to-glutamate ratios according to the 1q25 (rs10911021) genotype in $62 \mathrm{HUVECs} \mathrm{exposed} \mathrm{to} 5 \mathrm{mmol} / \mathrm{L}$ or $25 \mathrm{mmol} / \mathrm{L}$ glucose. Boxes correspond to the interquartile range, the line within the box to the median, and the cross to the mean. Whiskers correspond to the maximum and minimum. $B$ : Correlations of $\log _{2}$ GSH-to-glutamate ratio with $\log _{2} \gamma$-glutamyl-isoleucine and with $\log _{2}$ ophthalmate in $62 \mathrm{HUVECs}$ exposed to $5 \mathrm{mmol} / \mathrm{L}$ or $25 \mathrm{mmol} / \mathrm{L}$ glucose.

transfection in 14 different HUVEC strains. On average, GLUL protein levels were $43 \%$ lower $(P<0.0001)$ in cells transfected with a shRNA targeting GLUL than in the same cells transfected with a scrambled shRNA (Fig. 5B). Such reduction in GLUL levels was accompanied by a $40 \%$ increase in methylglyoxal levels $(P=0.03)$ (Fig. 5C). This was completely prevented $(P=0.0004)$ by exposing cells to increased concentrations of glutamine-the product of the enzymatic reaction catalyzed by GLUL (Fig. 5C).

\section{DISCUSSION}

In the current study, we used a targeted metabolomic approach to investigate the association between the 1q25
CHD locus and intracellular levels of 35 metabolites involved in glutamic acid metabolism and the $\gamma$-glutamyl cycle in 62 HUVEC strains naturally carrying different genotypes for the 1q25 lead variant rs10911021. This analysis was based on the hypothesis of an impairment of the $\gamma$-glutamyl cycle and/or glutamic acid metabolism as the mechanism linking the 1q25 locus to CHD, which was prompted by our previous finding of an association of the 1q25 risk allele with decreased expression of the nearby GLUL (glutamate-ammonia ligase) gene in endothelial cells and with a lower pyroglutamic-to-glutamic acid ratio in plasma (3). Our data, demonstrating differences among 1q25 genotypes in the cellular content of nine metabolites related to the $\gamma$-glutamyl cycle and glutamic acid metabolism 

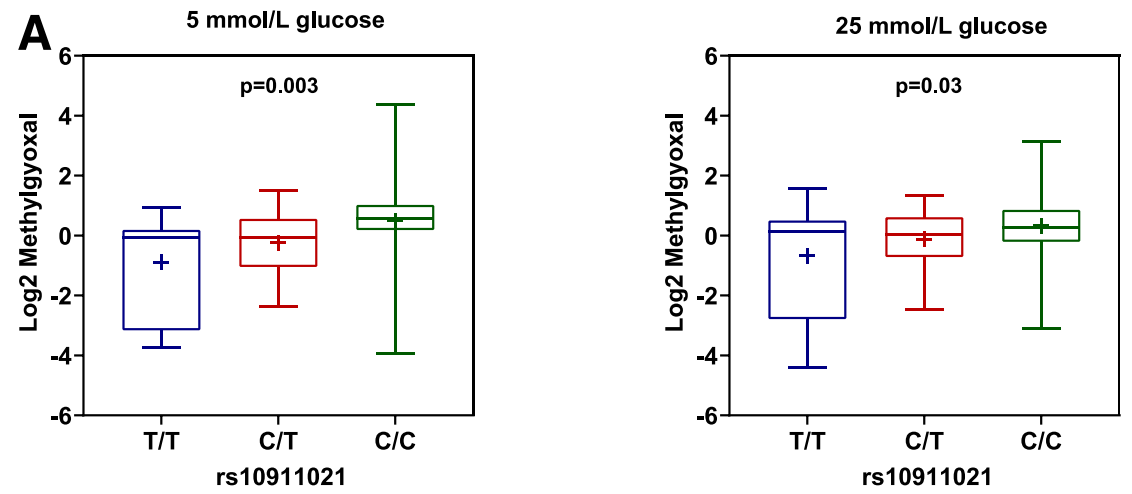

B

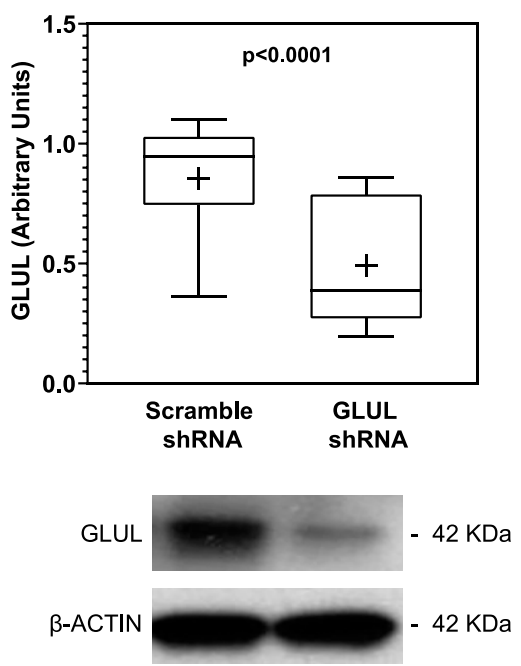

C

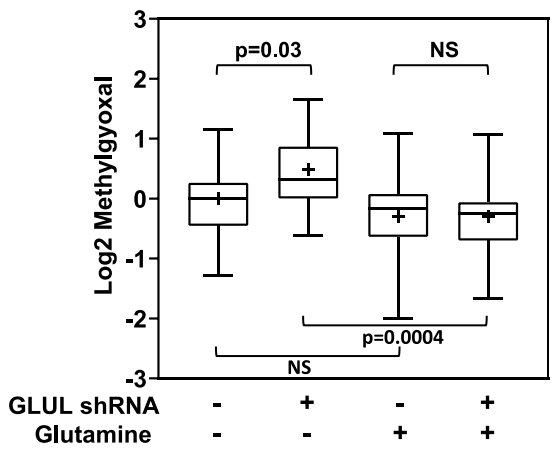

Figure 5-Methylglyoxal levels in HUVECs. A: Distributions of $\log _{2}$ levels of methylglyoxal (arbitrary units after normalization by assay batch) in 62 HUVECs exposed to $5 \mathrm{mmol} / \mathrm{L}$ or $25 \mathrm{mmol} / \mathrm{L}$ glucose. B: Distributions of GLUL protein levels (arbitrary units after normalization by $\beta$-actin signal intensity and assay batch) in 14 HUVECs transfected with scramble or GLUL shRNA. Representative Western blot bands are shown below the plot. C: Distributions of methylglyoxal levels (arbitrary units after normalization by assay batch) in 14 HUVECs transfected with scramble or GLUL shRNA, with or without glutamine supplementation. Boxes correspond to the interquartile range, the line within the box to the median, and the cross to the mean. Whiskers correspond to the maximum and minimum values.

and to the AGE precursor methylglyoxal, which is normally detoxified by GSH, support this hypothesis.

Of the nine metabolites displaying a significant association with the $1 \mathrm{q} 25$ variant, eight, including five $\gamma$-glutamyl amino acids, ophthalmate, $\beta$-citryl-glutamate, and $N$-acetyl-aspartyl-glutamate, had increased levels in HUVECs carrying the 1q25 risk allele. Since all of these metabolites include a glutamyl residue, their increased levels may indicate an imbalance between the increased availability of glutamic acid (resulting from the decreased activity of GLUL associated with the risk allele) and the availability of cysteine for the synthesis of $\gamma$-glutamylcysteine by glutamate cysteine ligase (GCL, the $\gamma$-glutamyl cycle rate-limiting enzyme). Under these conditions, the excess glutamic acid may be transferred to other amino acids by GCL, leading to increased synthesis of $\gamma$-glutamyl amino acids other than $\gamma$-glutamyl-cysteine (12) (Fig. 6). A similar mechanism can be hypothesized for the increased levels of ophthalmate - a tripeptide analog of GSH without its antioxidant properties-resulting from the GCL-mediated transfer of glutamic acid to L-2-aminobutyrate (rather than cysteine), followed by the addition of glycine (13) (Fig. 6). The increase in ophthalmate and other glutamylcontaining molecules, which is similar to that observed in situations of increased oxidative stress (14), suggests an inefficient utilization of glutamic acid toward the synthesis of GSH in endothelial cells carrying the 1q25 risk allele. Indeed, while GSH levels were not significantly different among 1q25 genotypes, the ratio between GSH and glutamate levels was inversely correlated to the risk allele dosage in both low and high glucose. Also, in both conditions, the GSH-to-glutamate ratio showed a highly significant inverse correlation with $\gamma$-glutamyl-isoleucine, $\gamma$-glutamyl-leucine, $\gamma$-glutamyl-valine, $\gamma$-glutamyl-threonine, and ophthalmate.

Given that the CHD-predisposing effect of the 1q25 locus found in previous studies was specific to people with diabetes (3), the finding of a similar association between 


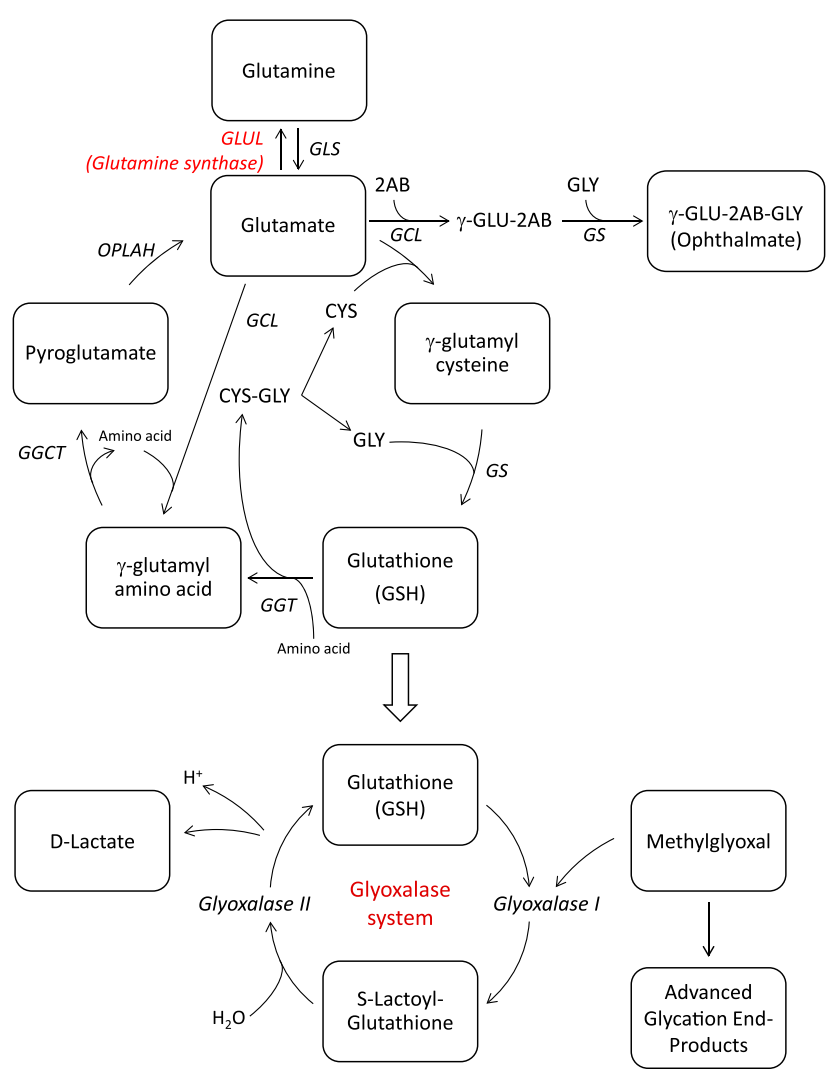

Figure 6-Schematic representation of the $\gamma$-glutamyl cycle and glyoxalase system.

genotype and intracellular metabolic features in low and high glucose (i.e., the lack of SNP $\times$ glucose interaction) deserves a comment. A possible explanation is that glucose exposure for $48 \mathrm{~h}$ did not adequately reproduce the effects of chronic exposure to high glucose occurring in people with diabetes. Alternatively, the interaction between 1q25 and the diabetic milieu on CHD risk observed in vivo may be mediated by other metabolic characteristics typical of type 2 diabetes, such as insulin-resistance and/or dyslipidemia (15), rather than hyperglycemia itself. The possible interaction of the 1q25 genotype with these additional diabetes-associated exposures will have to be investigated in further studies.

Alone among the metabolites associated with the 1q25 locus, $S$-lactoyl-glutathione showed a negative association with the 1q25 genotypes, its level being markedly reduced in cells homozygous for the risk allele. S-lactoyl-glutathione is mostly generated from glutathione in the glyoxalase system during the detoxification of methylglyoxal, which is a highly toxic by-product of glucose and lipid metabolism that has been implicated in the etiology of vascular complications of diabetes as a precursor of AGEs (11,16-19). Methylglyoxal reacts with GSH to form a thiohemiacetal (16), which is converted into $S$-lactoyl-glutathione by the enzyme glyoxalase 1 and then to lactate and glutathione by glyoxalase 2 (Fig. 6). Reduced $S$-lactoyl-glutathione has been associated with dysregulation of hepatic methylglyoxal detoxification in a mouse model of homocystinuria (14). Consistent with a dysregulation of methylglyoxal detoxification, we observed a striking increase in methylglyoxal (2.8-fold in low glucose and 2.1-fold in high glucose) in HUVECs homozygous for the risk allele compared with cells homozygous for the protective allele. Of note, GLUL downregulation through shRNA interference significantly increased methylglyoxal levels, supporting the hypothesis that the association between the 1q25 risk allele and methylglyoxal levels is sustained by a cause-andeffect relationship. The mechanism underlying this effect may relate to the inefficient synthesis of GSH described above, despite the apparent lack of significant differences in absolute GSH levels among 1q25 genotypes. This hypothesis is supported by our finding of another marker of oxidative stress (3-chloro-tyrosine) (20) being significantly associated with the risk allele in HUVECs exposed to high glucose as well as by a report in the literature describing an association of the 1q25 risk allele with lower plasma levels of GSH and higher levels of the oxidative stress biomarker malondialdehyde (21). Another possibility to explain the mechanism triggering the observed association is inhibition of glyoxylase 1 activity by some of the metabolites, such as $\gamma$-glutamyl amino acids or ophthalmate, that are increased in risk allele carriers and have been shown to impact cellular transporters and signaling $(13,22)$.

Important for translating our findings to develop new interventions to prevent CHD in diabetes, the increase in methylglyoxal levels associated with GLUL downregulation was prevented by increasing the culture medium concentration of glutamine five times above the levels normally used to preserve cell functions during prolonged periods of incubation (9). Glutamine is a known precursor of NAD, and oral supplementation of this amino acid has been shown to raise the NAD redox ratio in red blood cells from patients with sickle cell anemia (23) and to be associated with clinical improvement in the frequency of sickle cell crises in a phase 3 clinical trial (24). If the beneficial effect on methylglyoxal production by vascular cells is confirmed by further studies and is also demonstrated in animal models of atherosclerosis, glutamine supplementation may be considered as a candidate intervention for CHD prevention among individuals with type 2 diabetes who carry the 1q25 risk allele and have lower glutamine synthase activity.

Our study has several strengths, including the use of a large number of untransformed human primary cells, through which cellular functions could be studied without artifacts deriving from immortalization, the availability of cells from multiple subjects, allowing the study of naturally occurring genetic variants, without the need for genetic manipulation, and the systematic assessment of a large panel of metabolites involved in glutamic acid metabolism and the $\gamma$-glutamyl cycle.

However, some potential limitations must be acknowledged. While HUVECs have been extensively used for in vitro studies of the impact of diabetes on the endothelium 
$(25,26)$, their metabolic characteristics and response to stimuli may not perfectly capture those of endothelial cells lining the coronary arteries (27). Also, while our study provided an innovative snapshot of the metabolic differences between 1q25 genotypes based on the cell content of key metabolites, inferences made on the basis of these data will have to be corroborated by more detailed studies of metabolic fluxes and enzymatic activities. Finally, our study was focused on the metabolic traits associated with the 1q25 risk allele. However, we cannot exclude that nonmetabolic effects also contribute to the increased CHD risk associated with this locus as suggested by the recent report of an unexpected role of glutamate-ammonia ligase (the enzyme coded by GLUL) in angiogenesis (28). Also, we cannot exclude a role of changes in the expression of other genes, although GLUL was the only one of the eight genes in the 1q25 region analyzed in the original report for which a significant association was observed between mRNA levels and rs10911021 (3). Finally, no adjustment for multiple comparisons was made. While this was based on the prespecified nature of the analysis and on the high correlation among the metabolites included in this study, the possibility of false positives results cannot be excluded.

In summary, our findings in a unique collection of HUVEC strains from multiple individuals provide further support for an association between the diabetes-specific CHD locus at 1q25 and alterations of glutamic acid metabolism and the $\gamma$-glutamyl cycle. They also suggest decreased GSH-mediated detoxification of the AGE precursor methylglyoxal as a mechanism linking this locus to increased CHD risk and raise the hypothesis of glutamine supplementation as an approach to prevent CHD in carriers of this genetic risk factor.

Acknowledgments. The authors thank Dr. Pamela Di Tomo (Department of Medical, Oral and Biotechnological Sciences, Center for Advanced Studies and Technology - CAST (ex CeSI-MeT), University G. d'Annunzio of Chieti-Pescara, Chieti, Italy) for her skilled technical assistance in HUVEC harvesting and banking. Funding. This research was supported by a research grant from Sanofi (to A.D.), National Institutes of Health National Institute of Diabetes and Digestive and Kidney Diseases grant DK36836 (Molecular Phenotyping and Genotyping Core and Enrichment Core of the Diabetes Research Center at the Joslin Diabetes Center) and grants DK081943 and DK082841 (to S.Pe.), the Italian Ministry of Health (2018-2020 to S.Pr. and Ricerca Corrente 2019-2020 to V.T.), the Italian Ministry of University and Research (PRIN 2015 to V.T.), and Fondazione Roma (Grant for Biomedical Research: Non Communicable Diseases, Call for proposals 2013 to V.T.).

The content of the article is solely the responsibility of the authors and does not necessarily represent the official views of the National Institutes of Health or other funding entities.

Duality of Interest. A.D. reports a grant from Sanofi supporting the submitted work. No other potential conflicts of interest relevant to this article were reported.

Author Contributions. C.P. and A.D. designed the study, acquired, analyzed, and interpreted the data, wrote the initial draft of the manuscript, and revised the manuscript to its final form. H.S. analyzed and interpreted the data and revised the manuscript to its final form. S.Pr., V.T., and A.P. contributed to the study design, interpreted the data, and revised the manuscript to its final form.
N.D.P., L.Z., K.P., and S.Pe. acquired, analyzed, and interpreted the data and revised the manuscript to its final form. A.D. is the guarantor of this work and, as such, had full access to all the data in the study and takes responsibility for the integrity of the data and the accuracy of the data analysis.

Prior Presentation. Parts of this study were presented in poster form at the 79th Scientific Sessions of the American Diabetes Association, San Francisco, CA, 7-11 June 2019.

\section{References}

1. Fox CS, Coady S, Sorlie PD, et al. Increasing cardiovascular disease burden due to diabetes mellitus: the Framingham Heart Study. Circulation 2007;115: 1544-1550

2. Doria A. Leveraging genetics to improve cardiovascular health in diabetes: the 2018 Edwin Bierman Award Lecture. Diabetes 2019;68:479-489

3. Qi L, Qi Q, Prudente S, et al. Association between a genetic variant related to glutamic acid metabolism and coronary heart disease in individuals with type 2 diabetes. JAMA 2013;310:821-828

4. Look AHEAD Research Group. Prospective association of GLUL rs10911021 with cardiovascular morbidity and mortality among individuals with type 2 diabetes: the Look AHEAD study. Diabetes 2016;65:297-302

5. Prudente $\mathrm{S}$, Shah $\mathrm{H}$, Bailetti $\mathrm{D}$, et al. Genetic variant at the GLUL locus predicts all-cause mortality in patients with type 2 diabetes. Diabetes 2015;64: 2658-2663

6. Krebs HA. Metabolism of amino-acids: the synthesis of glutamine from glutamic acid and ammonia, and the enzymic hydrolysis of glutamine in animal tissues. Biochem J 1935;29:1951-1969

7. Reid M, Jahoor F. Glutathione in disease. Curr Opin Clin Nutr Metab Care 2001;4:65-71

8. Förstermann U, Xia N, Li H. Roles of vascular oxidative stress and nitric oxide in the pathogenesis of atherosclerosis. Circ Res 2017;120:713-735

9. Di Tomo P, Lanuti P, Di Pietro N, et al. Liraglutide mitigates TNF- $\alpha$ induced pro-atherogenic changes and microvesicle release in HUVEC from diabetic women. Diabetes Metab Res Rev 2017;33:e2925

10. Vivekanandan-Giri A, Byun J, Pennathur S. Quantitative analysis of amino acid oxidation markers by tandem mass spectrometry. Methods Enzymol 2011; 491:73-89

11. Thornalley PJ. Glyoxalase I--structure, function and a critical role in the enzymatic defence against glycation. Biochem Soc Trans 2003;31:1343-1348

12. Bachhawat AK, Yadav S. The glutathione cycle: glutathione metabolism beyond the $\gamma$-glutamyl cycle. IUBMB Life 2018;70:585-592

13. Dello SA, Neis EP, de Jong MC, et al. Systematic review of ophthalmate as a novel biomarker of hepatic glutathione depletion. Clin Nutr 2013;32:325330

14. Maclean KN, Jiang $\mathrm{H}$, Aivazidis $\mathrm{S}$, et al. Taurine treatment prevents derangement of the hepatic $\gamma$-glutamyl cycle and methylglyoxal metabolism in a mouse model of classical homocystinuria: regulatory crosstalk between thiol and sulfinic acid metabolism. FASEB J 2018;32:1265-1280

15. Warram JH, Kopczynski J, Janka HU, Krolewski AS. Epidemiology of noninsulin-dependent diabetes mellitus and its macrovascular complications. A basis for the development of cost-effective programs. Endocrinol Metab Clin North Am 1997;26:165-188

16. Maessen DE, Stehouwer CD, Schalkwijk CG. The role of methylglyoxal and the glyoxalase system in diabetes and other age-related diseases. Clin Sci (Lond) 2015;128:839-861

17. Schalkwijk CG, Stehouwer CDA. Methylglyoxal, a highly reactive dicarbonyl compound, in diabetes, its vascular complications, and other age-related diseases. Physiol Rev 2020;100:407-461

18. Hanssen NMJ, Scheijen JLJM, Jorsal A, et al. Higher plasma methylglyoxal levels are associated with incident cardiovascular disease in individuals with type 1 diabetes: a 12-year follow-up study. Diabetes 2017;66:2278-2283

19. van Eupen MG, Schram MT, Colhoun HM, et al. The methylglyoxal-derived AGE tetrahydropyrimidine is increased in plasma of individuals with type 1 diabetes 
mellitus and in atherosclerotic lesions and is associated with SVCAM-1. Diabetologia 2013;56:1845-1855

20. Shao B, Pennathur S, Heinecke JW. Myeloperoxidase targets apolipoprotein $A-I$, the major high density lipoprotein protein, for site-specific oxidation in human atherosclerotic lesions. J Biol Chem 2012;287:6375-6386

21. Shahid SU, Shabana, Humphries S. The SNP rs10911021 is associated with oxidative stress in coronary heart disease patients from Pakistan. Lipids Health Dis 2018;17:6

22. Broadhead GK, Mun HC, Avlani VA, et al. Allosteric modulation of the calciumsensing receptor by gamma-glutamyl peptides: inhibition of PTH secretion, suppression of intracellular cAMP levels, and a common mechanism of action with L-amino acids. J Biol Chem 2011;286:8786-8797

23. Niihara Y, Zerez CR, Akiyama DS, Tanaka KR. Oral L-glutamine therapy for sickle cell anemia: I. Subjective clinical improvement and favorable change in red cell NAD redox potential. Am J Hematol 1998;58:117-121
24. Niihara Y, Miller ST, Kanter J, et al.; Investigators of the Phase 3 Trial of LGlutamine in Sickle Cell Disease. A phase 3 trial of L-glutamine in sickle cell disease. N Engl J Med 2018;379:226-235

25. Lorenzi M, Cagliero E, Toledo S. Glucose toxicity for human endothelial cells in culture. Delayed replication, disturbed cell cycle, and accelerated death. Diabetes 1985;34:621-627

26. Castellano I, Di Tomo P, Di Pietro N, et al. Anti-inflammatory activity of marine ovothiol A in an in vitro model of endothelial dysfunction induced by hyperglycemia. Oxid Med Cell Longev 2018;2018:2087373

27. Garcia-Cardeña G, Comander J, Anderson KR, Blackman BR, Gimbrone MA Jr. Biomechanical activation of vascular endothelium as a determinant of its functional phenotype. Proc Natl Acad Sci U S A 2001;98:44784485

28. Eelen G, Dubois C, Cantelmo AR, et al. Role of glutamine synthetase in angiogenesis beyond glutamine synthesis. Nature 2018;561:63-69 